\section{$\underset{\substack{\text { hommes } \\ \text { \& migrations }}}{ }$}

\section{Hommes \& migrations}

Revue française de référence sur les dynamiques

migratoires

1319 | 2017

Réfugiés et migrants au Liban

\title{
Moh! Kouyaté
}

\section{François Bensignor}

Journals

\section{Édition électronique}

URL : http://journals.openedition.org/hommesmigrations/4011

DOI : ERREUR PDO dans /localdata/www-bin/Core/Core/Db/Db.class.php L.34 : SQLSTATE[HY000]

[2006] MySQL server has gone away

ISSN : 2262-3353

Éditeur

Musée national de l'histoire de l'immigration

\section{Édition imprimée}

Date de publication : 1 octobre 2017

Pagination : 175-179

ISBN : 978-2-919040-39-1

ISSN : 1142-852X

Référence électronique

François Bensignor, « Moh! Kouyaté », Hommes \& migrations [En ligne], 1319 | 2017, mis en ligne le 01 octobre 2017, consulté le 08 janvier 2021. URL : http://journals.openedition.org/hommesmigrations/ 4011 ; DOI : https://doi.org/10.4000/hommesmigrations.4011 


\section{MUSIQUE}

\section{MOH ! KOUYATÉ}

Propos recueillis par FRANÇOIS BENSIGNOR.

Citoyen du monde par son statut de musicien et Parisien de cœur, Moh ! Kouyaté partage sa vision d'une nouvelle Afrique de son point de vue de Guinéen. L'art du continent dont se nourrit son œuvre est connecté à toute la planète. Il en a l'expérience par ses tournées américaines, européennes. Alors qu'il s'est d'abord forgé un nom comme guitariste et orchestrateur auprès de nombreux artistes, un premier album remarqué en 2015, Loundo (Un jour), lui permet de donner près de 200 concerts avec son propre groupe. Le titre de son nouvel album Fé Toki (Point de vue), paru au printemps 2017, laisse entendre que tout est question de point de vue... Retraçons avec lui son parcours artistique.

$A$ îné d'une famille de djéli (griots) de Conakry, la ville où il est né le 21 avril 1977, Moh! Kouyaté garde des attaches fortes avec la capitale guinéenne et sa culture traditionnelle. Paris, où il est installé depuis 2007, lui sert de base pour développer une carrière internationale pleine de promesse. Dans son appartement parisien du $X^{e}$ arrondissement, rentré d'un long séjour à Conakry, il évoque ses débuts: "Dès l'école primaire, après la classe, nous commencions déjà à taper sur des palmes de cocotiers. Puis, vers l'âge de sept ans, mon papa m'a appris les bases du balafon.»

Moh ! va accompagner son père dans les mariages et les baptêmes qu'animent les djéli par tradition. Le balafon étant l'apanage des familles Kouyaté, c'est l'instrument avec lequel le fils aîné devra se familiariser. Et il perfectionnera son jeu aux côtés de ses oncles maternels, djéli virtuoses du balafon.
Modjigui Kouyaté, le grand-père paternel de Moh !, un djéli malinké, a installé sa famille dans le quartier de Hafia, majoritairement habité par des Diankhanké. Sa mère appartenant à l'ethnie soussou, le garçon va maîtriser trois langues dès l'enfance (le soussou, le malinké et le diakhanké), qu'aujourd'hui il utilise indifféremment dans ses chansons.

\section{Du balafon à la guitare}

Si un djéli Kouyaté se doit de jouer du balafon, l'instrument de prédilection de son grand-père comme de son père est la guitare. À 12 ans, il reçoit un ukulélé des mains de sa grand-mère paternelle qu'il adore et chez laquelle, enfant, il avait l'habitude de manger et de dormir. Alors qu'il fait jouer ses doigts sur les quatre cordes de la petite guitare, 


\section{MUSIQUE}

l'aïeule incite le garçon à chanter, car c'est aussi la tradition chez les djéli que de savoir vocaliser harmonieusement la parole.

Deux ans plus tard, voulant tâter de la six cordes de son père, il en casse une malencontreusement. Au lieu des représailles auxquelles il s'attend, Moh! va bénéficier de ses premiers enseignements à la guitare, dispensés par son père avec bienveillance. À 16 ans, il trouve en Amadou Sadio Diallo un maître guitariste qui lui permet non seulement de perfectionner son jeu, mais aussi de s'ouvrir à d'autres musiques: "Un jouril ma donné une cassette de Georges Benson. Je n'y comprenais rien mais j'étais subjugué. » Des amis musiciens et des Français expatriés contribueront à enrichir ses connaissances musicales en lui faisant découvrir Jimi Hendrix, B. B. King, Django Reinhardt... Depuis, sa passion pour la guitare ne se dément pas.

\section{L'émulation de Conakry}

Dans les années 1990 à Conakry, une grande émulation s'exerce sur les jeunes musiciens issus des familles de djéli. L'une d'elles, autour du patriarche joueur de kora M'Bady Kouyaté, est particulièrement dynamique. "Nos deux familles se fréquentaient et travaillaient ensemble, donc les enfants aussi » se rappelle Moh !. II va former un groupe avec le neveu et les deux fils du patriarche, Ba Cissoko et ses cousins Abdoulaye et Kourou Kouyaté. Ensemble, ils animent les fêtes privées des expatriés européens et les fameuses soirées dansantes de l'hôtel Camayenne, sur la corniche. Leur répertoire est fait de reprises de chansons à la mode, de succès du répertoire mandingue moderne, parsemé de quelques créations.

Enthousiasmé par ces débuts instructifs, le jeune guitariste décide de fonder avec ses frères sa propre formation, Conakry Cocktail. Il assume parfaitement son rôle d'aîné, transmettant son savoir musical non seulement aux membres de sa famille, mais aussi à d'autres jeunes qui viennent le voir afin d'apprendre la musique. À 20 ans, l'avenir lui sourit, et il s'entend avec son groupe pour faire danser les clubs de Conakry comme le Loft ou La Fourchette Magique. "À l'époque, on vivait notre passion. On voulait s'exprimer avecl'envie de donner tout ce qu'on avait. "

\section{Des guitaristes pour modèles}

À cette époque, quelques-unes des anciennes grandes formations d'État, voulues et financées par le président guinéen Sékou Touré, étaient encore en activité, comme le mythique Syli Authentic. "La nuit, j'allais voir en cachette l'orchestre Kegne Star à l'autre bout de la ville, se souvient Moh !. J'étais curieux, jeécoutais tout et je fréquentais beaucoup de groupes. Je suis aussi allé rencontrer directement de grands guitaristes qui m'impressionnent, comme Sékou Diabaté du Bembeya Jazz. Je l'ai revu plusieurs fois depuis mes débuts et il m'a laissé entendre qu'il apprécie ce que je fais. On ne peut pas parler de la guitare africaine sans évoquer le nom de Sékou "Bembeya", surnommé "Diamond Fingers", qui a inventé et incarne toujours le style de la guitare moderne guinéenne."

La Guinée a produit d'immenses musiciens et notamment des guitaristes, dont de nombreux enregistrements témoignent. Ceux du groupe Virtuoses Diabaté, qui rassemble trois frères, I'aîné « Docteur » Sékou Diabaté, et deux plus jeunes, Abdoulaye et Siré, préfigurent l'indépassable African Virtuoses, fondé en 1979 par les deux premiers, constitué de quatre guitaristes acoustiques et d'un percussionniste, qui forgent une musique à la douceur d'une exclusive intemporalité. "I'ai beaucoup écouté Ousmane Kouyaté, l'ancien guitariste des Ambassadeurs Internationaux, qui accompagne encore souvent Salif Keïta. II a beaucoup influencé mon jeu de guitare » explique Moh!, évoquant également Sékou Kora Kouyaté, Sékou Kélétigui ou Petit Condé, entre autres guitaristes de talent qui ont formé son oreille. 
et guitariste peuhl, Doura Barry a traversé les premières décennies de l'indépendance au sein de formations guinéennes prestigieuses, dont le Syli Authentic, le Kaloum Star ou le Camayenne Sofa, avant de consolider sa notoriété sous son seul nom. Moh! l'accompagne en France, en Belgique et en Suisse à l'occasion du $12^{\mathrm{e}}$ Festival Francophonie Métissée organisé par le Centre Wallonie-Bruxelles.

\section{Rencontre avec le blues}

De retour au pays, sa rencontre avec Corey Harris sera déterminante pour sa carrière. Invité à Conakry en 2004 par l'ambassade des États-Unis, le bluesman américain y donne une "master class », à laquelle Moh! participe. Le courant passe si bien entre les deux hommes qu'en 2006 Corey lui demande d'in-

tégrer son groupe à l'occasion d'une tournée américaine de trois mois. À 29 ans, le guitariste guinéen parcourt l'est des États-Unis, depuis la presqu'île de Key West, à l'extrême pointe de la Floride, jusque dans le Vermont, près de la frontière avec le Canada. "Nous jouions dans des clubs et dans des festivals, raconte-t-il. La voiture nous emmenait d'une ville à l'autre. Je découvrais des paysages nouveaux, des lieux dédiés à la musique dont je n'avais pas idée. Cette expérience restera l'un de mes plus beaux souvenirs de tournée."

"Key West, qui se trouve à $200 \mathrm{~km}$ de la côte cubaine, m'a particulièrement impressionné, autant que le vermont où il régnait un froid polaire. Bien sûr, New York m'a fait grande impression. En tournant aux États-Unis, j'ai eu l'occasion de rencontrer des musiciens noirs américains. Dans ce milieu du blues, je ne me sentais pas vraiment dépaysé. Même si je ne comprenais pas l'anglais, j'ai retrouvé dans leur attitude, leurs manières de faire, beaucoup de points communs avec l'Afrique: une spontanéité, une façon de rire, de ressentir la musique, qui m'étaient familières. 


\section{MUSIQUE}

"Quand le public venait me voir après le concert, il s'étonnait que je ne parle pas sa langue. Alors que je jouais leur musique, ils ne comprenaient pas quand je leur disais que je venais d'Afrique... Un jour, dans une université du Mississippi où il n'y avait que des Noirs, je présentais le balafon. Les étudiants, qui ne connaissent pas l'Afrique, me posaient des questions incongrues. Ils pensaient que les Africains vivent toujours sur le mode traditionnel, dans des villages de huttes, etc. Ils n'imaginaient pas qu'il puisse y avoir de grands centres urbains, les nouvelles technologies... Alors que Corey jouait les interprètes en français, ils demandaient si nous parlions "africain"..." "

Sur le chemin du retour vers la Guinée, Moh ! fait escale à Paris où il a de nombreuses relations musicales. Il y fait la rencontre de celle qui va devenir sa femme. En 2007, une nouvelle tournée avec Corey Harris l'amène en Europe et, à la fin de cette année, Moh !, âgé de 30 ans, se marie et s'installe à Paris.

\section{Vivre à Paris}

Dans cette ville d'art cosmopolite, Moh! se sent bien. Il va se faire un nom dans le milieu des musiciens. À la faveur de jam sessions dans les clubs du XVIII ${ }^{e}$ arrondissement où se retrouvent les meilleurs - Hilaire Penda, Étienne M’Bappé, Mokhtar Samba, etc. -, le guitariste se fait connaître par le bouche-à-oreille. S'ensuivent diverses collaborations, comme avec le jazzman Antoine Hervé, mais aussi avec ces anciens compagnons de route africains, Ba Cissoko et la chanteuse Fatoumata Diawara.

En 2005, sur le tournage du film de Cheick Fantamady Camara, II va pleuvoir sur Conakry, où la jeune actrice/chanteuse malienne jouait l'un des premiers rôles, le courant était passé avec Moh ! qui y faisait une apparition. Ils se retrouvent à Paris où la jeune actrice est décidée à lancer sa carrière de chanteuse. Pour s'accompagner,

Pochette de l'album Fé Toki de Moh! Kouyaté (Foli Son Productions, 2017). @ D. R.

Fatoumata Diawara a besoin d'apprendre la guitare. Moh! sera son professeur, avant de devenir son guitariste et orchestrateur dans la réalisation de son premier album, puis sur les scènes de ses nombreuses tournées.

\section{Enregistrer sous son nom}

Si son talent de guitariste permet à Moh! d'évoluer auprès d'artistes de renom, il lui reste à affirmer, depuis l'Europe, celui d'auteur-compositeurinterprète. C'est chose faite avec Loundo (Un jour), premier album paru en 2015. Favorablement accueilli par les médias, ce disque autoproduit entraîne une série de près de 200 concerts à travers le monde. Une belle réussite qui l'encourage à publier un second album, Fé Toki (Point de vue) en 2017.

"À présent, je vis plus souvent à Paris qu'à Conakry, mais je suis Guinéen dans l'âme, dit Moh !. En voyageant et en vivant hors de mon pays, j'apprends beaucoup de choses. Mais les bases qui me constituent sont celles de la culture guinéenne. Parfois, dans une chanson, je vais essayer de toucher un peu plus mon public parisien ou celui, très cosmopolite, qui m'écoute et me regarde sur Internet. Je chante beaucoup plus en soussou, en malinké ou en 
diakhanké, parce que j'ai une facilité à m'exprimer dans ces langues. Sij'avais cette même facilitéà le faire en français ou en anglais, je le ferais. Quoi qu'il en soit, j'essaye toujours de m'adresser à un large public. »

\section{Question de point de vue}

"Dans l'album Fé Toki, j'ai envie de faire passer certains messages. "Vivons de l'Amour", avec ses paroles en français, est plutôt un regard sur ce que le monde vit aujourd'hui. II y a trop de haine, trop de racisme. Je pense aux hommes politiques, mais aussi à l'attitude des policiers envers le peuple. Des gens demandent ce que les Noirs font icien France. Un vieux discours raciste revient, contre lequel il faut faire passer des messages d'ouverture. Dans la société de nos parents, de nos aînés, le djéli a pour rôle d'adresser les messages à l'égard du peuple. Je suis un djéli et j'assume cet héritage. Ce n'est pas parce qu'il vient d'une tradition ancienne qu'il faut s'en débarrasser. Je suis partisan de donner un coup de modernité à cette tradition, dont les valeurs ne s'effacent pas. Un des rôles des djéli, depuis qu'ils existent dans la société mandingue, demeure celui de sensibiliser la société et de tempérer entre les différents points de vue.

"Chacune de mes chansons porte un message à travers une histoire que je raconte. Parmi les thèmes forts de l'album, "Tala" (Le partage) évoque l'actualité brûlante et déchirante des jeunes qui veulent coûte que coûte partir de leur pays pour changer leur destin. Veulent-ils seulement faire l'expérience de ce qui peut leur arriver? Peut-être rêvent-ils juste

de façonner leur vie dans un sens positif. Certains partent de Libye et périssent en mer... C'est le moment d'interpeller l'Europe et les Européens afin qu'ils comprennent bien que ces migrants sont des humains dénués de mauvaises intentions. Ils ont juste besoin de changer de destin pour acquérir une vie meilleure. Je dis que le partage n'est pas équitable. Ceux qui ont les moyens pensent-ils à tous ces jeunes qui se sentent abandonnés, errant sur les routes en se cherchant eux-mêmes ? Ont-ils réfléchi àce qu'ils peuvent faire pour les aider un peu? II suffit parfois de peu pour redonner espoir et faire changer les choses." . 